\title{
Multiple Myeloma presenting as Gingival mass
}

\author{
Ajaz Shah $\cdot$ Ashaq Ali $\cdot$ Suhail Latoo $\cdot$ \\ Irshad Ahmad
}

Received: 25 March 2009/Accepted: 18 April 2010/Published online: 22 September 2010

(C) Association of Oral and Maxillofacial Surgeons of India 2010

\begin{abstract}
Maxillofacial presentations in patients with multiple myeloma are not uncommon, but multiple myeloma is often overlooked. Because the symptoms are various, it is very difficult to diagnose multiple myeloma in the oral and maxillofacial region. On the basis of the pathologic and clinical findings, the patient was diagnosed with multiple myeloma. Hematologic screening and a radiographic survey further confirmed the diagnosis.
\end{abstract}

Keywords Multiple myeloma .

Punched-out osteolytic lesions .

Monoclonal gamma globulins

\section{Introduction}

Multiple myeloma is a malignant disease characterized by multifocal proliferation of atypical plasma cells and, in most patients, by the presence of monoclonal gamma globulins and/or their subunits, in the serum often referred to as " $\mathrm{M}$ " or myeloma proteins [1]. The incidence of the oral lesions in multiple myeloma varies from less than 2-70\% [1, 2]. Bruce and Royer [3] and Miller et al. [4] reported that $20-30 \%$ of all cases of multiple myeloma showed jaw involvement. Epstein et al. [5] reviewed 783 patients in the literature and indicated that approximately

A. Shah $(\bowtie) \cdot$ A. Ali $\cdot$ I. Ahmad

Department of Oral and Maxillofacial Surgery,

Government Dental College and Hospital, Srinagar,

Jammu and Kashmir, India

e-mail: drajazshah@gmail.com

S. Latoo

Department of Oral and Maxillofacial Pathology,

Government Dental College and Hospital, Srinagar, India
$14 \%$ of patients had oral manifestations. Oral lesions rarely occur as the first sign of the disease [6-10]. The common clinical signs and symptoms of multiple myeloma include pain in the bone, fatigue, anemia and infectious diseases. Radiography frequently shows the typical 'punched-out' osteolytic lesions $[6,8,11]$. We report a case of multiple myeloma in the oral cavity presenting as a right sided maxillary gingival/alveolar mass with hypermobility of the adjacent teeth.

\section{Case Report}

A 52-year-old man presented with a chief complaint of right sided maxillary gingival/alveolar mass with hypermobility of the adjacent teeth for 10 days. The patient had undergone extraction of right maxillary 2nd molar 15 days back. Upon intraoral examination, a soft, non tender, non-ulcerated, non-hemorrhagic tissue mass $(1.5 \times 2 \times 2 \mathrm{~cm})$ arising from extraction socket of right maxillary 2 nd molar was seen. The lesion was extending from right 1 st maxillary molar to tuberosity region. Right maxillary first molar had grade 2 mobility (Fig. 1). Panoramic and PNS radiography showed an osteolytic lesion in the right posterior maxilla body and haziness of maxillary sinus.

After the radiographic evaluation, an incisional biopsy was taken from the right sided maxillary region. The histopathological investigation of the specimen revealed atypical plasma cells with large hyperchromatic nuclei and large areas of cytoplasm, and some of the cells were binucleated. Significant mitotic activity was detected in the tumour cells and there were condensed eosinophilic aggregations (Russell bodies) between the cells (Fig. 2). The histopathological diagnosis was plasma cell myeloma 


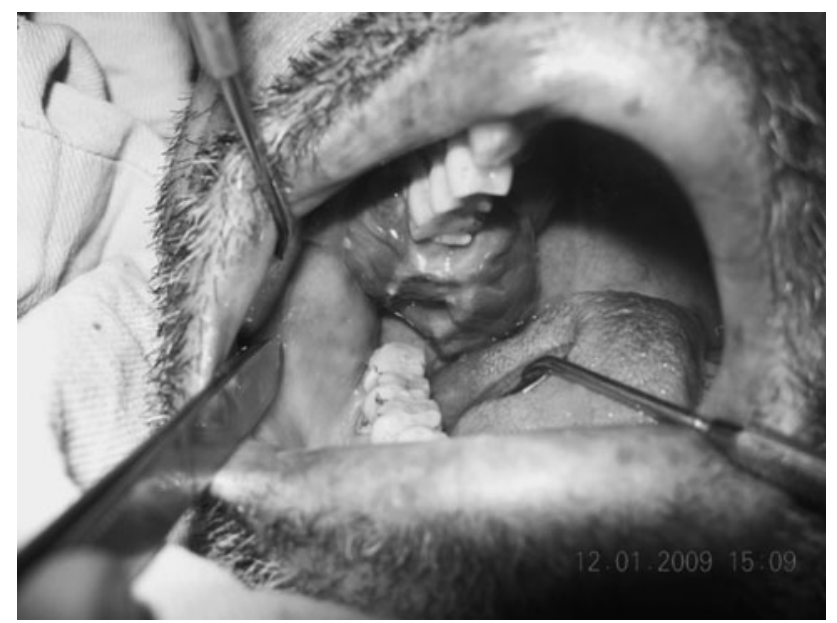

Fig. 1 Intraoral picture of myeloma

and the pathologist advised further investigation for multiple myeloma.

Skeletal radiographic survey of the skull and other bones revealed multiple 'punched-out' osteolytic lesions (Fig. 3). The urine was negative for Bence-Jones protein.

The patient was referred to an oncologist for further evaluation and appropriate treatment planning. The final diagnosis was confirmed multiple myeloma with serum protein immunoelectrophoresis and bone marrow biopsy. Bone marrow biopsy showed bone marrow specimens with nearly $100 \%$ cellularity. Especially in one intertrabecular area, almost all of the cells were comprised of atypical plasma cells with eccentric nuclei and eosinophilic cytoplasms. Mononuclear cells and megakaryocytes were also seen in the other areas. Immunohistochemical analysis revealed monoclonal IgG staining with kappa chain (Fig. 4). Appropriate chemotherapy (melphalan and prednisone) was initiated immediately. One month after the initial diagnosis patient developed spontaneous femur fracture and was managed in an orthopedic hospital.
The patient received palliative radiotherapy 5 months after initial diagnosis because of distant metastasis. Two months after radiotherapy patient rejected further therapy and died 5 months later, 7 months after the initial diagnosis.

\section{Discussion}

Plasma cell myeloma (plasmacytoma) may present as one of three distinct clinical entities: multiple myeloma, solitary plasmacytoma of bone, and extramedullary plasmacytoma [11]. Multiple myeloma is the multifocal disseminated form and is a malignant neoplastic condition characterized by the proliferation of a single clone of abnormal plasma cells [5]. Secondary invasion of the skeletal tissue is one of the most important characteristics of the disease [1]. Myelomatous infiltrates commonly involve the calvaria and mandible, pelvis, sternum, clavicle and proximal portions of the humerus and femur [12]. Multiple myeloma is most common in patients older than 40 years of age with a peak incidence rate at 60-70 years [6].

Maxillofacial presentations in patients with multiple myeloma are not uncommon, but multiple myeloma is often overlooked. The incidence of the oral lesions in multiple myeloma varies from less than $2-70 \%[1,2]$. Bruce and Royer [3] and Miller et al. [4] reported that $20-30 \%$ of all cases of multiple myeloma showed jaw involvement. Epstein et al. [5] reviewed 783 patients in the literature and indicated that approximately $14 \%$ of patients had oral manifestations. Oral lesions rarely occur as the first sign of the disease [6-10]. Jaw lesions may be the primary manifestation of multiple myeloma with an incidence varying from 8 to $15 \%$ [3,13]. Because the symptoms are various, it is very difficult to diagnose multiple myeloma in the oral and maxillofacial region. These include swelling, mass formation paresthesia of the lower lip, pain, bleeding and fracture of the jawbone, tooth
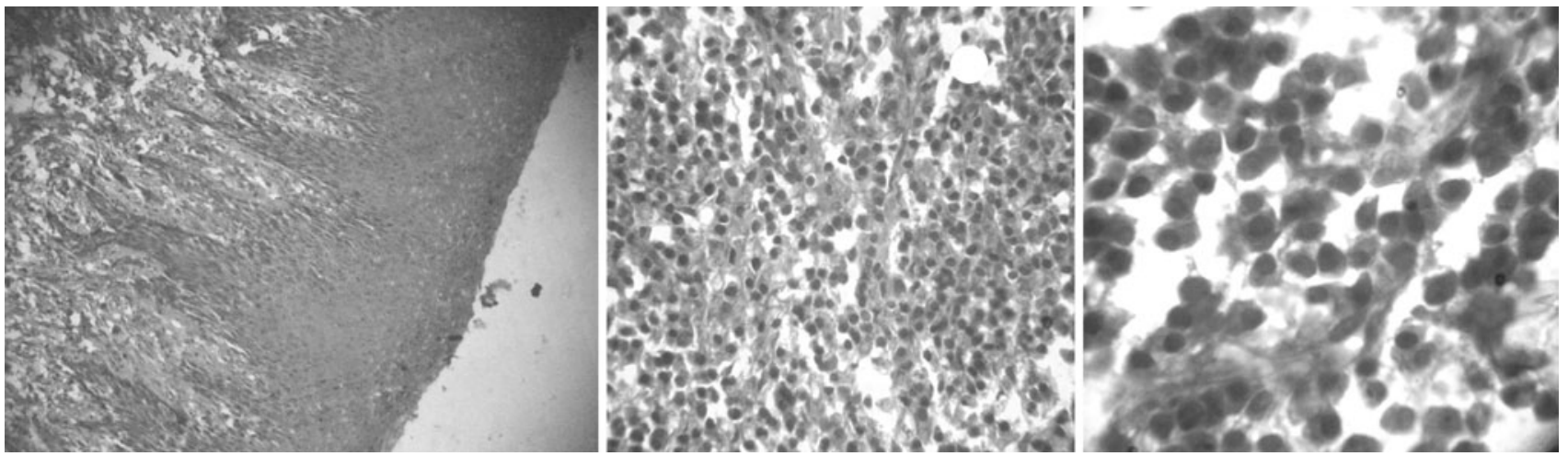

Fig. 2 Histopathological pictures showing diffusely infiltrating pleomorphic plasma cells 
Fig. 3 Radiographs revealing multiple punched-out osteolytic lesions
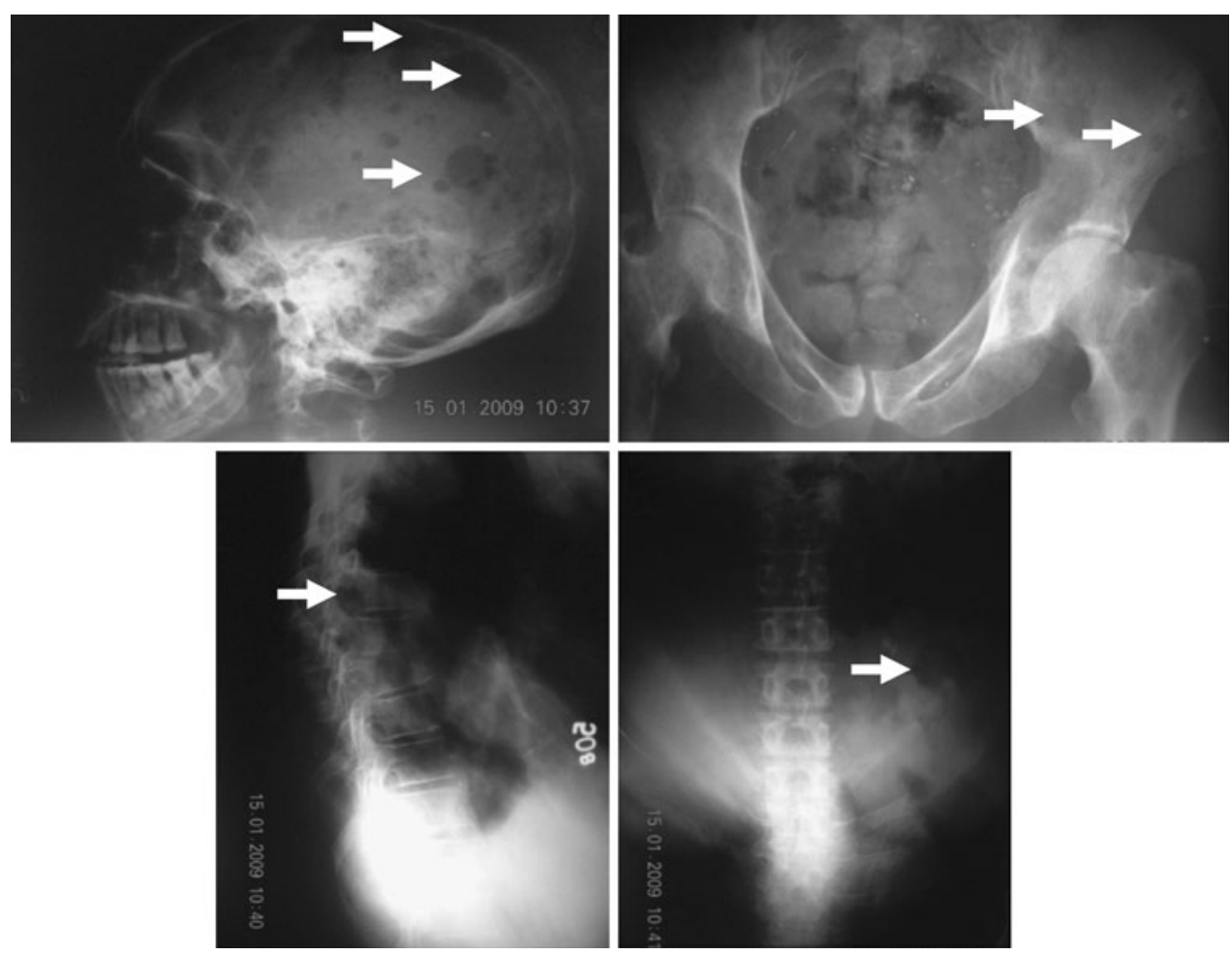

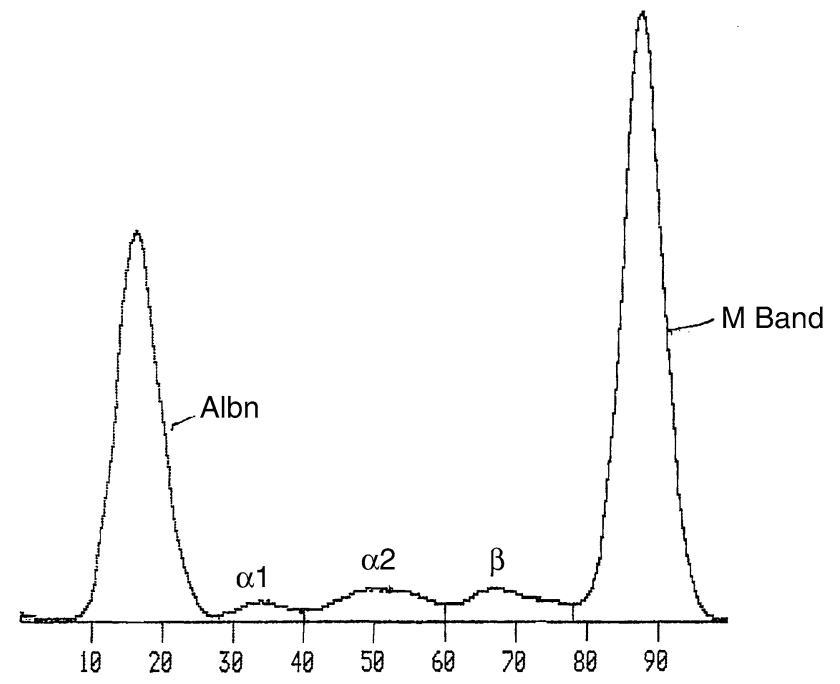

Fig. 4 Immunoelectrophoresis of serum protein revealing a monoclonal IgG with a chain

mobility and migration, macroglossia and radiolucent lesions $[8,14,15]$. Paresthesia, radiolucent bony lesions, pain and swelling are the commonly associated symptoms with multiple myeloma $[1,11,14]$. Osteolytic lesions are more frequent in the mandible than in the maxilla, especially in the posterior teeth region, ramus and condylar process, presumably because of greater hematopoietic activity in these areas [5, 11, 16]. In our case, a soft, non tender, non-ulcerated, non-haemorhagic tissue mass
$(1.5 \times 2.2 \mathrm{~cm})$ arising from extraction socket of right maxillary 2nd molar, was seen. Panoramic and PNS radiography showed an osteolytic lesion in the right posterior maxilla body and haziness of maxillary sinus.

The differential diagnosis of small, multiple, separate, well-defined radiolucencies includes multiple myeloma, multiple metastatic lesions and Langerhans' cell disease. When multiple punched-out, bony lesions are found in children, Langerhans' cell disease and multiple metastatic lesions are the most likely diagnoses. In adults, multiple myeloma and metastatic carcinoma are highly probable when several bones in the skeleton are involved. Many of the signs and symptoms of these two entities are similar, and therefore the clinical manifestations are not often beneficial in helping differentiate between them. Since multiple myeloma is more common than multiple metastatic disease, it should appear higher on the differential diagnosis [17]. Differential diagnosis of multiple myeloma from multiple metastatic lesions necessitates further clinical and laboratory evaluation, including histopathological examination [6]. If multiple myeloma is suspected, a multidisciplinary approach towards the diagnosis should always be followed. A full blood evaluation with differential and blood counts, biochemical assessment of renal function, calcium status, serum protein electrophoresis, quantification of immunoglobulins, immunoelectrophoresis, bone marrow biopsy and aspiration, urine analysis which includes Immunofixation Electrophoresis (IFE) and a radiographic skeleton survey should be obtained [12]. 
Suspect, asymptomatic, multiple, (and usually relatively small) radiolucencies in the jaws of an apparently healthy adult in whom no abnormalities have been disclosed by a general systemic and radiographic examination most likely represent multiple, distinct bone marrow defects [17]. Multiple myeloma should be distinguished from solitary plasmacytoma of bone. The criteria essential for the diagnosis of solitary plasmacytoma of bone are that the diagnosis should be established by biopsy, the skeletal survey should eliminate the presence of other foci, findings in bone marrow biopsy should be negative, there should be no dysproteinaemia or Bence-Jones proteinuria [18]. In our case, skeletal radiographic survey of the skull and other bones showed the presence of multiple oesteolytic lesions and later, bone marrow biopsy and laboratory examinations confirmed the diagnosis of multiple myeloma.

\section{Conclusion}

Maxillofacial manifestation in patients with multiple myeloma is not uncommon, but multiple myeloma is often overlooked. Therefore this disease should be borne in mind as a differential diagnosis when considering a patient whose chief complaint is unusual gingival/alveolar mass.

\section{References}

1. Lambertenghi-Deliliers G, Bruno E, Cortelezzi A, Fumagalli L, Morosini A (1988) Incidence of jaw lesions in 193 patients with multiple myeloma. Oral Surg Oral Med Oral Pathol 65(5): 533-537

2. Witt C, Borges AC, Klein K, Neumann HJ (1997) Radiographic manifestations of multiple myeloma in the mandible: a retrospective study of 77 patients. J Oral Maxillofac Surg 55(5): 450-453

3. Bruce KW, Royer RQ (1953) Multiple myeloma occurring in the jaws; a study of 17 cases. Oral Surg Oral Med Oral Pathol 6(6):729-744
4. Miller CD, Goltry RR, Shenasky JH (1969) Multiple myeloma involving the mandible. Report of a case. Oral Surg Oral Med Oral Pathol 28(4):603-609

5. Epstein JB, Emerton S, Guglietta A, Le N (1997) Assessment of epidermal growth factor in oral secretions of patients receiving radiation therapy for cancer. Oral Oncol 33(5):359-363

6. Mozaffari E, Mupparapu M, Otis L (2002) Undiagnosed multiple myeloma causing extensive dental bleeding: report of a case and review. Oral Surg Oral Med Oral Pathol Oral Radiol Endod 94(4):448-453

7. Lee SH, Huang JJ, Pan WL, Chan CP (1996) Gingival mass as the primary manifestation of multiple myeloma: report of two cases. Oral Surg Oral Med Oral Pathol Oral Radiol Endod 82(1):75-79

8. Epstein JB, Voss NJ, Stevenson-Moore P (1984) Maxillofacial manifestations of multiple myeloma. An unusual case and review of the literature. Oral Surg Oral Med Oral Pathol 57(3):267-271

9. Monje F, Gil-Diez JL, Campano FJ, Alonso del Hoyo JR (1989) Mandibular lesions as the first evidence of multiple myeloma. Case report. J Craniomaxillofac Surg 17(7):315-317

10. Furutani M, Ohnishi M, Tanaka Y (1994) Mandibular involvement in patients with multiple myeloma. J Oral Maxillofac Surg 52(1):23-25

11. Pisano JJ, Coupland R, Chen SY, Miller AS (1997) Plasmacytoma of the oral cavity and jaws: a clinicopathologic study of 13 cases. Oral Surg Oral Med Oral Pathol Oral Radiol Endod 83(2):265-271

12. Raubenheimer EJ, Lello GE, Dauth J, Fayman MS, Dvornak N, Senekal JC (1988) Multiple myeloma presenting as localized expansile jaw tumour. Int J Oral Maxillofac Surg 17(6):382-385

13. Zachriades N, Papanicolaou S, Papavassiliou D, Vairaktaris E, Triantafyllou D, Mezitis M (1987) Plasma cell myeloma of the jaws. Int J Oral Maxillofac Surg 16(4):510-515

14. Vincent SD, Lilly GE, Hupp JR (1993) Paresthesia of the mandibular division, trigeminal nerve. J Oral Maxillofac Surg 51(5):565-569

15. Ziegler CM, Flechtenmacher C, Mühling J (2002) Tender preauricular swelling. Oral Surg Oral Med Oral Pathol Oral Radiol Endod 93(5):516-520

16. Raubenheimer EJ, Dauth J, van Wilpe E (1987) Multiple myeloma: a study of 10 cases. J Oral Pathol 16(8):383-388

17. Wood NK, Goaz PW, Jacobs MC (1997) Multiple separate, welldefined radiolucencies. In: Wood NK, Goaz PW (eds) Differential diagnosis of oral and maxillofacial lesions, 5th edn. MosbyYear Book, Inc., St. Louis, pp 387-389

18. Loh HS (1984) A retrospective evaluation of 23 reported cases of solitary plasmacytoma of the mandible, with an additional case report. Br J Oral Maxillofac Surg 22(3):216-224 